\title{
Die Sache mit dem (Hirn-)Tod
}

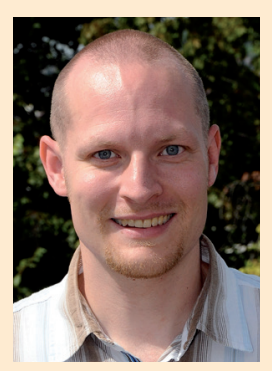

Christian Schäfer, Stuttgart
Der Deutsche Ethikrat ist sich nicht einig in der Sache, ob „hirntot sein“ gleich „tot sein“ ist. Dies hat er Ende Februar kommuniziert. Diese Uneinigkeit ist nicht verwunderlich, denn die Biologie des Menschen lässt sich eben nur bedingt in Schubladen einordnen, die wir des Verständnisses wegen so gerne anwenden: Es ist viel leichter zu verstehen, wenn man sagt „ab hier ist der Mensch tot“ und „ab dort lebt er noch“, oder? Aber wo ist diese Grenze nun - wenn das Gehirn so stark und irreversibel geschädigt ist, dass es „tot“ ist, und grundlegende Funktionen sowie das Bewusstsein daher nicht wiederkehren können, oder wenn das Herz nicht mehr schlägt, oder ...?

Wenn man sich auf die Ebene der Zellen begibt (die kleinste Einheit dessen, was wir zweifelsfrei als irdisches Leben bezeichnen), bemerkt man, dass keine Pauschalaussage möglich ist, wann Zellen z.B. nach einem Herzstillstand sterben: Denn die Zellen der verschiedenen Organe und Gewebetypen sterben nicht gleichzeitig, sondern nacheinander mit teilweise großen Zeitabständen. Der Übergang in den Tod ist auf dieser Ebene somit fließend (analog), und kein digitaler (plötzliche Änderung des „Zustandes Leben“ in den „Zustand Tod“). Er ist erst dann vollbracht, wenn die letzte lebende Zelle des Körpers tot ist. Und selbst dann bestünden noch Zweifel, denn z.B. im menschlichen Darm existiert eine vielfältige und lebensnotwendige Flora, die aus Bakterien besteht. Diese wird als Darmmikrobiom bezeichnet und von Mikrobiomforschern als eigenes Organ angesehen. Und wenn dieses beim Tod seines „Wirtes“ nicht gleich mitstirbt, ist der Mensch dann schon tot?

Würde man sich bzgl. der Organentnahme einer Definition des Todes bedienen, die besagt, dass jede einzelne Körperzelle gestorben sein muss, müsste man lange mit dem Beginn warten - so lange, dass man niemals eine Transplantation der z.B. infrage kommenden Nieren in Erwägung ziehen könnte. Die Organe wären hierfür unbrauchbar, weil alle Zellen irreversibel aus dem Leben geschieden sind. Man sieht sofort das Problem, wollen wir als Gesellschaft, dass Organspenden von z. B. (hirn-)toten Unfallopfern möglich sind. In Deutschland gilt die Dead-Donor-Rule - nur einem zweifelsfrei für tot erklärten Menschen darf man nach der Berücksichtigung des Patientenwillens bzw. der Entscheidung der Angehörigen Organe entnehmen (eine Ausnahme ist bekanntlich die Lebendspende, was aber hier nichts zur Sache tut).

In Deutschland wird bzgl. der Organentnahme der Gesamthirntod als zu erfüllendes Kriterium verwendet. Ein durch moderne und diverse Verfahren bestätigter Hirntod hat sich als sichere Diagnose erwiesen - zumindest hat laut den Aussagen von Ärzten noch niemand spontan (Teile seiner) Hirnfunktionen oder gar das Bewusstsein wieder erlangt, bei dem einmal der Hirntod nach strikten Kriterien der Bundesärztekammer (BÄK) festgestellt wurde. Das ist aus physiologischen Gründen sehr gut nachvollziehbar.

Auch der Deutsche Ethikrat erkennt dies an und empfiehlt, am diagnostizierten Hirntod für eine Legitimation der Entnahme von Organen festzuhalten. Er grenzt sich in seiner Stellungnahme vom 24. Februar auch deutlich von der Non-Heart-beating-Donation ab, wie sie in der Schweiz praktiziert wird: „Nach derzeitigen Erkenntnissen lässt eine lediglich fünf- bis zehnminütige Wartezeit nach Herzstillstand, wie sie im Ausland weit verbreitet ist, nicht den sicheren Schluss auf das irreversible Erlöschen aller Hirnfunktionen zu.“ Ich finde die Empfehlungen des Deutschen Ethikrates sehr gut, zumal zusätzlich auf die Notwendigkeit hingewiesen wird, die Informationsmaterialien für die Bürger zu diesem komplexen Thema dringend zu verbessern.

Mit der Möglichkeit des Todes muss man sich auch leider bei Intensivpatienten auseinandersetzen. Das akute Nierenversagen ist aufgrund der lebensbedrohlichen Situation und dem nephrologischen Aspekt ein großes Thema für Sie, liebe Leser. Daher haben die Gasteditorin Prof. Christiane Erley, Berlin, und ich zusammen den Schwerpunkt dieser Ausgabe der Dialyse aktuell gestaltet: „Intensivmedizin und Nephrologie“. Ich wünsche Ihnen eine angenehme Lektüre der interessanten Artikel ab Seite 138, für die wir wie gewohnt kompetente Autoren gewinnen konnten! 